\title{
Marcadores bioquímicos de estrés oxidativo en jugadoras universitarias de voleibol. Efecto del consumo de Passiflora edulis \\ Biochemical markers of oxidative stress in female volleyball players. Effect of consumption of Passiflora edulis \\ *Diana María García-Cardona, *Patricia Landázuri, **Carlos Federico Ayala-Zuluaga, *Beatriz Restrepo Cortes \\ *Universidad del Quindío (Colombia), **Universidad de Caldas (Colombia)
}

Resumen. Objetivo: examinar el efecto del consumo de Passiflora edulis (P. edulis) sobre marcadores bioquímicos de estrés oxidativo (BMEOX) en mujeres voleibolistas de una selección universitaria. Método:población catorce jugadoras (edad: 19.3 \pm 2.2 años; masa: $57.5 \pm 5.0 \mathrm{~kg}$; estatura: $1.62 \pm 6.2 \mathrm{~m}$ ), fueron divididas aleatoriamente en grupo experimental (GE) y grupo placebo (GP). Durante los siete días del microciclo, al GE se les suministró $2 \mathrm{ml} /$ día de zumo de P edulis y el GP recibió $2 \mathrm{ml} /$ día de placebo (agua saborizada). Se tomaron muestras sanguíneas durante los días 1, 4, 7 y 14. Se cuantificaron creatina quinasa (CK), glutamato oxalacetato transaminasa (GOT), glutamato piruvato transaminasa (GPT), creatinina (Cr), catalasa (CAT), superóxido dismutasa (SOD), la peroxidación lipídica por el método de sustancias reactivas al ácido tiobarbitúrico (TBARS) y la tasa de filtración glomerular (TFG). Resultados: las transaminasas, se incrementaron significativamente en el día 4, y descendieron en los días 7 y 14 en ambos grupos. CK y Cr, aumentaron, pero no retornaron a valores basales en el día 14 . TBARS y CAT aumentaron en ambos grupos sin diferencias significativas, mientras SOD aumentó con diferencias significativas para todos los días solo en GP. Conclusión: El microciclo produce cambios en los BMEOX, aumentando su nivel con el aumento de la intensidad del mismo; El consumo de P. edulis no afectó de manera significativa las actividades transaminasas, SOD y CAT, pero estas enzimas permanecen más estables en las deportistas que consumieron P.edulis. Es necesario profundizar el conocimiento sobre usos de antioxidantes y ejercicio intenso.

Palabras clave: voleibol, biomarcadores, estrés oxidativo, antioxidante, Passiflora edulis.

Abstract. Objective: to examine the effect of the consumption of Passiflora edulis (P. edulis) on biochemical markers of oxidative stress (BMEOX) in female volleyball players of a university team. Method: population fourteen players (age: $19.3 \pm$ 2.2 years; mass: $57.5 \pm 5.0 \mathrm{~kg}$; height: $1.62 \pm 6.2 \mathrm{~m}$ ), were randomly divided into experimental group (EG) and placebo group (GP). During the seven days of the microcycle, the EG was given $2 \mathrm{ml} /$ day of P edulis juice and the GP received $2 \mathrm{ml}$ / day of placebo (flavored water). Blood samples were taken during days 1, 4, 7 and 14. Creatine kinase (CK), glutamate oxaloacetate transaminase (GOT), glutamate pyruvate transaminase (GPT), creatinine (Cr), catalase (CAT), superoxide dismutase (SOD), lipid peroxidation by thiobarbituric acid reactive substances (TBARS) and glomerular filtration rate (GFR). Results: transaminases increased significantly on day 4, and decreased on days 7 and 14 in both groups. CK and Cr, increased, but did not return to baseline values on day 14. TBARS and CAT increased in both groups without significant differences, while SOD increased with significant differences for all days only in GP. Conclusion: The microcycle produces changes in the BMEOX, its level increase with the increase in its intensity; Consumption of P. edulis did not significantly affect transaminase, SOD and CAT activities, but these enzymes remain more stable in athletes who consumed P. edulis. It is necessary to deepen the knowledge about antioxidant uses and intense exercise.

Key words: volleyball, biomarkers, oxidative stress, antioxidant, Passiflora edulis.

\section{Introducción}

El voleibol es un deporte que se desarrolla en equipo y es considerado según la clasificación, como un deporte de pelota (Bouet, 1968), de equipo (Matveiev, 1975) y/o cooperación-oposición (Blázquez \& Hernández, 1984); dentro del juego, se presentan infi-

Fecha recepción: 09-03-20. Fecha de aceptación: 04-08-21

Diana María García-Cardona

dmgarcia@uniquindio.edu.co nidad de acciones e intervenciones inciertas por parte de los jugadores, estas pueden ser cortas o largas; en el voleibol moderno se presentan acciones tácticas muy rápidas que duran entre 4 y 40 segundos, aunque en promedio son 8 segundos, y que no permiten la recuperación rápida del organismo, además en el juego se busca definir rápidamente los puntos del partido con eficientes y adecuadas ejecutorias (Martínez-Rodríguez et al., 2017; Rodríguez, 2015; Vitón, 2012).

Las acciones durante el juego de voleibol se evidencian en saltos, desplazamientos variados, rápidos y ex- 
plosivos (potencia y resoluciones de ejecución) (SánchezMoreno et al., 2018) mediante la aplicación de fuerza rápida o explosiva-balística, presentándose regímenes de exigencias muscular, cardiacas y respiratorias altas, pero especialmente el sistema energético anaerobio alactácido, lo que genera ciertos tipos de estrés afectando el equilibrio homeostático de los jugadores durante el juego, requiriendo participaciones cortas, frecuentes, de alta intensidad y con períodos de baja intensidad y breves descansos (Picón-Martínez et al., 2019; Rodriguez, 2015; Vilela et al., 2021).

Al respecto existe una relación directa en las demandas del entrenamiento deportivo y el estrés, así, a mayor exigencia de rendimiento deportivo mayores los niveles de estrés (García-Cardona, Landázuri \& SánchezMuñoz, 2021; Molinero, Salguero \& Márquez, 2012; Reynoso-Sánchez, Hernández-Cruz, López-Walle, Rangel-Colmenero, Quezada-Chacón \& Sánchez, 2016). Durante el entrenamiento físico intenso, las demandas de energía a nivel muscular aumentan la capacidad oxidativa del miocito, y con ello una mayor producción de especies reactivas de oxígeno y radicales libres (Azizbeigi, Stannard, Atashak, \& Mosalman, 2013), lo que conduce a una exacerbada respuesta inflamatoria (Fernández, Da Silva-Grigoletto \&Túnez-Fiñana, 2009), y por consiguiente al padecimiento de excesivo dolor y fatiga muscular posterior al ejercicio (Reid, 2008); sin embargo, el entrenamiento crónico conduce a respuestas de adaptación de los diferentes sistemas, y presupone un cambio también en variables bioquímicas y fisiológicas, es así, como al parecer se regulan al alza las enzimas de defensa antioxidante, las cuales interfieren con los sistemas oxidativos para la eliminación y reparación de daños (Radak, Chung \& Goto, 2008).

La adaptación de los sistemas fisiológicos al estrés oxidativo generado por el entrenamiento puede ser apoyada en positivo, por la ingesta de nutrientes antioxidantes, los cuales fortalecen los sistemas de defensa antioxidantes endógenos de los deportistas, contrarrestando los efectos negativos del daño oxidativo debido a los radicales libres (Buonocore \& Negro, 2015). Se han identificado propiedades protectoras en sustancias consideradas como antioxidantes, entre las que se incluyen vitaminas, polifenoles, selenio, entre otros, por lo tanto, una adecuada alimentación o suplementación podrá contribuir a disminuir los productos derivados del estrés oxidativo generados por el ejercicio.

$\mathrm{Al}$ respecto hay evidencia creciente en relación con la utilización de antioxidantes naturales como coadyuvantes en deportistas, por ejemplo (Estrada-Díaz,
Hernández-Cruz, Cruz-Castruita, Zavala-Flores, Rodríguez-Bies \& Rangel-Colmenero, 2017) encontraron que la ingesta de zarzamora (Rubus sp.), rica en antioxidantes es favorable en el entrenamiento previo a la competencia ya que promueve la regulación del estrés oxidativo, disminuyendo los valores del mismo; también los arándanos (Vaccinium corymbosum), han demostrado beneficios potenciales en la regulación del estrés oxidativo inducido por ejercicio (Nieman et al., 2013), Martins et al (Martins et al., 2020) mostró que la ingesta de zumo de jugo de uva (Cooperativa Nova Aliança, RS) reconstituido a partir de la especie Vitis labrusca durante dos semanas parece reducir la oxidación de proteínas y el daño del ADN por el ejercicio físico.

Una de las plantas con alto contenido de sustancias antioxidantes es la P. edulis, planta conocida con el nombre común de maracuyá, parchita, maracujá, yellow passion-fruit (García, 2002); esta es cultivada ampliamente en regiones tropicales y subtropicales en varias partes del mundo, especialmente en América del Sur, el Caribe, el sur de Florida, Sudáfrica y Asia (Hu et al., 2017). El fruto de P. edulis mide entre 6 y $12 \mathrm{~cm}$ de largo y entre 4 y $7 \mathrm{~cm}$ de diámetro, la cáscara es de color amarillo brillante, dura y espesa, las semillas son marrones, la pulpa es ácida y tiene un fuerte sabor aromático (He et al., 2020). La P. edulis ha sido utilizada tanto para el consumo humano como en la medicina tradicional; dentro de los usos en la gastronomía, este fruto puede ser utilizado tanto en cocina fría, caliente y repostería. Se consume el fruto ya sea fresco o en jugos, helados, dulces, tortas, salsas, entre otras. Con respecto a los usos medicinales la $P$. edulis es relajante, puede utilizarse como ligero sedante, además posee efecto antihipertensivo (Guerrero-Ospina et al., 2018), entre otros.

Dentro de los componentes funcionales de la P.edulis se incluyen polifenoles, proteínas, flavonoides, alcaloides, compuestos cianogénicos, glucósidos, vitaminas, minerales y compuestos terpenoides (Gunathilake, Ranaweera \& Rupasinghe, 2018; Rotta, Rodrigues, Cristina, Fontes, Maldaner \& Visentainer, 2019; SeptembreMalaterre, Stanislas, Douraguia \& Gonthier, 2016; Zeraik et al., 2011).

Existen diversos estudios del efecto del consumo de Passiflora edulis en mujeres no deportistas, por ejemplo, con sobrepeso y obesidad (Dolores et al., 2018) en donde encontraron que la ingesta de una bebida a base de Physalis peruviana, Passiflora edulis, Ananas comosus, Avena sativa, Linum usitatissimum y Stevia rebaudiana, tuvo efecto hipolipemiante en los niveles séricos de triglicéridos. 
Otro estudio evaluó clínicamente los efectos del extracto de semilla de maracuyá rico en piceatannol en la piel de mujeres japonesas sanas (Maruki-Uchida et al., 2018), encontrando aumentos significativos en el contenido de humedad de la piel en comparación con antes del ensayo, además, los resultados mostraron reducciones significativas en la transpiración y fatiga en el grupo que consumió el extracto de semilla de maracuyá en comparación con el grupo placebo. En el estudio (Kitada et al., 2017) en donde investigaron los efectos del piceatannol (purificado a partir de extracto de semilla de Passiflora edulis) sobre la salud metabólica en humanos, encontraron que la suplementación con piceatannol mejora la sensibilidad a la insulina y podría reducir la presión arterial y la frecuencia cardiaca en hombres con sobrepeso, pero no en mujeres con y sin sobrepeso.

Muchos de los componentes que posee P.edulis, tales como los polifenoles han sido estudiados en relación con su efecto en diferentes marcadores bioquímicos y fisiológicos (Guerrero-Ospina et al., 2018; Silva et al., 2015; Zhang et al., 2016), sin embargo, el efecto del consumo de jugo $P$ edulis, sobre marcadores bioquímicos en atletas mujeres de la disciplina de voleibol, no ha sido estudiado, a pesar que estudios como el de (González \& Rivas, 2018), mencionan que las diferencias femeninas en la respuesta fisiológica al ejercicio están relacionadas con el tamaño corporal, la composición corporal y la endocrinología reproductiva.

Dada la información anterior, el propósito de este estudio fue examinar el efecto del consumo de Passiflora edulis sobre marcadores bioquímicos de estrés durante un microciclo que simulaba la competencia deportiva en mujeres voleibolistas de una selección universitaria.

\section{Material y método}

Se diseñó un estudio experimental de corte longitudinal, tipo ensayo comunitario, con tamaño de muestra por conveniencia.

Participaron 14 voleibolistas de la selección femenina de la Universidad del Quindío (Colombia), con experiencia en torneos nacionales, y cuya práctica deportiva incluía sesiones de entrenamiento de 10 a 14 horas por semana como parte de su preparación para torneos regionales y nacionales.

El método de reclutamiento de los sujetos de estudio consistió en invitar a las deportistas a una reunión informativa, en donde se dio a conocer del proyecto los objetivos, metodología, riesgos, beneficios, y se solucionaron inquietudes.
Se excluyeron del estudio las deportistas con enfermedad comprobada a través de su historia clínica. También se excluyeron las deportistas lesionadas, y que llevaran menos de un año de entrenamiento en la disciplina deportiva.

La investigación se realizó de acuerdo con la Declaración de Helsinki y la Resolución 8430 del Ministerio de Salud y Protección Social de Colombia. El proyecto fue avalado por el Comité de Bioética (Acta No 01 del 5 febrero de 2018) del Programa de Educación Física de la Universidad del Quindío. Las deportistas voluntariamente firmaron el consentimiento informado en el cual se especificó su libertad para abandonar la investigación si así lo decidieran.

\section{Procedimiento}

Las deportistas se distribuyeron aleatoriamente (ciego simple), siete en el grupo experimental (GE) y siete en el grupo placebo (GP). Las voleibolistas participaron en un microciclo de entrenamiento de siete días.

\section{Suministro de antioxidante}

Durante los siete días del microciclo, el GE consumió $2 \mathrm{ml}$ / día de zumo de Passiflora edulis, $(1 \mathrm{~g} / \mathrm{ml})$, GP ingirió $2 \mathrm{ml}$ de placebo, el cual estaba preparado en agua natural purificada con colorante vegetal amarillo. El consumo de la bebida para ambos grupos fue de una dosis diaria por la mañana antes de cada entrenamiento durante 7 días. La dosis de zumo fue tomada de estudios previos de Rojas et al 2009, y Guerrero-Ospina et al 2018) en pacientes hipertensos.

A las deportistas se les solicitó abstenerse del consumo de maracuyá, así como de alimentos ricos en antioxidantes.

\section{Microciclo}

El microciclo fue implementado en el periodo preparatorio (etapa especial), ya cercano al periodo competitivo. Este microciclo se desarrolló bajo la simulación de la competencia (zonal universitario) donde las atletas debían desempeñarse durante cinco partidos a la mayor capacidad de rendimiento posible en cada uno de estos. A continuación, se muestran las características del microciclo:

Día 1: Trote suave, movimiento articular, activación muscular, estiramiento activo

Día 2: Entrenamiento táctico

Día 3: Partido 1

Día 4: Partido 2

Día 5: Partido 3 
Día 6: Partido 4

Día 7: Partido 5

Un total de cinco partidos fueron disputados durante el período experimental, los partidos tuvieron una duración entre 90 y 120 minutos distribuidos en 5 sets.

A las jugadoras se les mostró la escala de esfuerzo percibido de Borg al finalizar cada sesión de entrenamiento. Todos los sujetos estaban familiarizados con esta escala, como parte del seguimiento regular de la formación.

\section{Toma de muestra}

La muestra sanguínea fue recolectada por punción venosa en tubo seco, el suero se obtuvo por centrifugación a 1000 g por 15 minutos, a $4{ }^{\circ} \mathrm{C}$, separado en microtubos de 1,5 mL y almacenado en refrigerador hasta su uso (dentro de 2 días).

Se tomaron cuatro muestras de sangre de la siguiente manera:

Día 1 (D1): La muestra sanguínea fue recolectada después de 48 horas sin ejercicio y doce horas de ayuno.

Día 4 (D4) y 7 (D7): La muestra sanguínea fue recolectada una vez terminado el entrenamiento.

Día 14 (D14): Siete días después de terminado el microciclo. La muestra sanguínea fue recolectada después de 48 horas sin ejercicio y doce horas de ayuno.

Durante los cinco días posteriores al último partido, las deportistas entraron a un microciclo de restablecimiento, en donde realizaron descanso activo (alrededor del $40 \%$ de la frecuencia cardiaca máxima).

\section{Mediciones bioquímicas}

Las mediciones bioquímicas se realizaron así: la creatina quinasa (CK), glutamato oxalacetato transaminasa (GOT), glutamato piruvato transaminasa (GPT) y creatinina (Cr) fueron cuantificadas por métodos enzimáticos colorimétricos (Wiener Lab®, Rosario, Argentina. CK: catálogo 870340022. GOT: catálogo 870580000 . GPT: catálogo 870600000. Cr: catálogo 864115024), la catalasa (CAT) y superóxido dismutasa (SOD) fueron cuantificados por métodos enzimáticos colorimétricos (Invitrogen $\AA^{\circledR}$, Frederick, USA. CAT: catálogo EIACATC. SOD: catálogo EIASODC), con lectura en el espectrofotómetro (génesis 5) a $450 \mathrm{~nm}$ para SOD y lectura en el espectrofotómetro (Epoch) a 560 nm para CAT, siguiendo las instrucciones del fabricante (BioLegend $囚)$, la peroxidación lipídica se cuantifico por el método de sustancias reactivas al ácido tiobarbitúrico (TBARS).

La tasa de filtración glomerular (TFG) fue calculada a través de la fórmula de Cockcroft-Gault ajustado por superficie corporal.

\section{Valoración antropométrica}

Antes de realizar las valoraciones antropométricas se siguieron las indicaciones de la International Society for the Avancement in Kineanthropometric (ISAK), se procedió al marcaje de los puntos anatómicos de referencia necesarios para la obtención de las medidas a estudiar, utilizando un lápiz dermográfico. Los puntos anatómicos marcados se encontraron en las siguientes posiciones: acromial, radial, estiloideo, iliocrestal, ilioespinal, trocantéreo, tibial, ángulo infraescapular y abdominal lateral. En todos los casos, las marcaciones fueron realizadas al lado derecho del sujeto. Las mediciones se tomaron partiendo de la posición antropométrica de referencia: masa, talla, talla sentada, pliegues cutáneos (tricipital, subescapular, supraespinal, abdominal, muslo y pierna) y perímetros (brazo relajado, antebrazo, muslo 1 máximo, pierna, torácico, cefálico y cintura).

\section{Análisis estadístico}

Para cada una de las variables analizadas se calculó la media y la desviación estándar (DE).

Se aplicó el test de Kruskal-Wallis.

También se realizó modelamiento de la media, a través del análisis de perfil de respuesta, ya que lo que se buscaba era caracterizar los patrones de cambio en la respuesta media a lo largo del tiempo en los grupos (GP y GE) y determinar si las formas en la media del perfil de respuesta diferían entre el grupo. Para el análisis de los datos se utilizó el programa R.

\section{Resultados}

Los resultados presentados en la Tabla 1, corresponden a la media más o menos la desviación estándar de la edad y las variables antropométricas de los sujetos de estudio. En esta, se observa que no se presentaron diferencia estadística entre GE y GP. Con respecto al porcentaje adiposo y muscular, se aprecia que, en promedio, ambos grupos se encuentran dentro de los estándares adecuados para edad y deporte.

\begin{tabular}{|c|c|c|c|}
\hline Variable & GE & GP & $p$-valor \\
\hline Edad (años) & $19(2.7)$ & $19.6(1.8)$ & .610 \\
\hline Masa (kg) & $58(4.43)$ & $57(8.1)$ & .908 \\
\hline Estatura (m) & $1.62(6.7)$ & $1.62(6.1)$ & .989 \\
\hline$\%$ Adiposo & $16.6(3.6)$ & $16.15(2.8)$ & .852 \\
\hline$\%$ Muscular & $41.40(2.6)$ & $43.92(3.1)$ & .326 \\
\hline
\end{tabular}




\begin{tabular}{|c|c|c|c|c|c|c|c|c|c|c|c|}
\hline \multirow{2}{*}{ Variable } & \multicolumn{4}{|c|}{ GE } & \multicolumn{4}{|c|}{ GP } & \multicolumn{3}{|c|}{$p$-valor } \\
\hline & D1 & D4 & D7 & D14 & D1 & D4 & D7 & D14 & Días & GE & GP \\
\hline \multirow{4}{*}{ GPT (U/L) } & \multirow{4}{*}{$12.09(2.8)$} & \multirow{4}{*}{$16.88(3.54)$} & \multirow{4}{*}{$14.17(3,27)$} & \multirow{4}{*}{$13.07(3.24)$} & \multirow{4}{*}{$10.32(.55)$} & \multirow{4}{*}{$13.14(1.82)$} & \multirow{4}{*}{$11.7(1.06)$} & \multirow{4}{*}{$10.77(2.51)$} & D1 vs D4 & .016 & .004 \\
\hline & & & & & & & & & D4 vs D7 & .150 & .79 \\
\hline & & & & & & & & & D7 vs D14 & .536 & .296 \\
\hline & & & & & & & & & D1 vs D14 & .724 & .850 \\
\hline \multirow{4}{*}{$\begin{array}{l}\text { GOT } \\
(\mathrm{U} / \mathrm{L})\end{array}$} & \multirow{4}{*}{$16.33(2.1)$} & \multirow{4}{*}{$22.24(3.25)$} & \multirow{4}{*}{$18.83(3.87)$} & \multirow{4}{*}{$16.68(2.99)$} & \multirow{4}{*}{$11.44(1.93)$} & \multirow{4}{*}{$18.18(2.67)$} & \multirow{4}{*}{$15.75(1.70)$} & \multirow{4}{*}{$13.85(2.30)$} & D1 vs D4 & .006 & .009 \\
\hline & & & & & & & & & D4 vs D7 & .142 & .335 \\
\hline & & & & & & & & & D7 vs D14 & .281 & .290 \\
\hline & & & & & & & & & D1 vs D14 & .975 & .885 \\
\hline \multirow{4}{*}{$\begin{array}{c}\text { CK } \\
\text { (U/L) }\end{array}$} & \multirow{4}{*}{$37.19(7.0)$} & \multirow{4}{*}{$160.73(48.3)$} & \multirow{4}{*}{$151.09(44.07)$} & \multirow{4}{*}{$62.76(20.3)$} & \multirow{4}{*}{$38.38(9.84)$} & \multirow{4}{*}{150.01} & \multirow{4}{*}{ ) $144.41(26.1)$} & \multirow{4}{*}{67.01} & D1 vs D4 & .001 & .001 \\
\hline & & & & & & & & & D4 vs D7 & .851 & .614 \\
\hline & & & & & & & & & D7 vs D14 & .001 & .019 \\
\hline & & & & & & & & & D1 vs D14 & .037 & .024 \\
\hline \multirow{4}{*}{$\mathrm{Cr}(\mathrm{mg} / \mathrm{dl})$} & & & & & & & & & D1 vs D4 & .003 & .016 \\
\hline & & & & & & & & & D4 vs D7 & .925 & .698 \\
\hline & $.88(.05)$ & $1.14(.18)$ & $1.15(.27)$ & $.91(.06)$ & $.88(.06)$ & $1.14(.28)$ & $1.23(.26)$ & $.92(.08)$ & D7 vs D14 & .039 & .032 \\
\hline & & & & & & & & & D1 vs D14 & .418 & .546 \\
\hline & & & & & & & & & D1 vs D4 & .002 & .036 \\
\hline 1FG & & & & & $98.38(7.23)$ & $7997(16.5)$ & $7379(15-5)$ & 95728 & D4 vs D7 & .944 & .645 \\
\hline$(\mathrm{mL} / \mathrm{mmn} /$ & & & & $93.54(8.10)$ & $98.38(7.23)$ & $19.97(16.3)$ & $13.1 /(15.5)$ & $93.12(12.6)$ & D7 vs D14 & .029 & .031 \\
\hline & & & & & & & & & D1 vs D14 & .402 & .717 \\
\hline
\end{tabular}

en el día 4 con respecto al día 1 . Mientras que esta enzima presentó una variabilidad significativa en cada día en GP. TBARS presentó el mismo comportamiento tanto en GE como en GP, es decir, con significancia estadística el incremento del día 4 con respecto al día 1 , y $\sin$

Los resultados presentados en la Tabla 2, corresponden a la media más o menos la desviación estándar de las variables bioquímicas evaluadas en los cuatro días, en esta se aprecia que las transaminasas (GOT y GPT), presentaron comportamiento similar en ambos grupos, incrementándose significativamente en el día 4 con respecto al día 1 , y con posterior descenso en los días 7 y 14 (sin significancia estadística entre los dos días). Siete días después de terminado el microciclo la $\mathrm{CK}$, en ambos grupos (GE y GP) no retornó a valores basales del día $1(p<.05)$. también se presentaron cambios significativos en la concentración de creatinina con un incremento en el día 4 con respecto al día 1 , y una disminución en el día 7 comparado con el día 14. Con respecto a los marcadores de estrés oxidativo (SOD, CAT y TBARS) no se encontraron diferencias significativas al comparar el GE con el GP (Tabla 3).

Tabla 3

\begin{tabular}{ccccc} 
Marcadores de estrés oxidativo & \multicolumn{5}{l}{} \\
\hline \multicolumn{1}{c}{ Variable } & Día & GE & GP & $p$-valor \\
\hline & 1 & $2.78(.61)$ & $2.85(.52)$ & .087 \\
SOD $(\mathrm{U} / \mathrm{mLs})$ & 4 & $4.03(.28)$ & $4.19(.30)$ & .110 \\
& 7 & $3.68(.35)$ & $3.75(.32)$ & .970 \\
& 14 & $3.12(.62)$ & $3.1(.60)$ & .271 \\
& 1 & $23.14(1.59)$ & $23.84(.34)$ & .570 \\
CAT $(\mathrm{U} / \mathrm{mL})$ & 4 & $24.27(.79)$ & $24.70(.39)$ & .330 \\
& 7 & $24.25(.74)$ & $24.33(.37)$ & .682 \\
& 14 & $23.95(1.09)$ & $24.29(.38)$ & .343 \\
& 1 & $8.49(2.14)$ & $8.05(2.5)$ & .811 \\
TBARS (mmol/L) & 4 & $12.33(2.48)$ & $13.51(3.35)$ & .076 \\
& 7 & $10.31(2.49)$ & $12.04(3.6)$ & .809 \\
& 14 & $9.11(2.0)$ & $9.32(2.2)$ & .708 \\
\hline Media (desviación estándar). SOD: superóxido dismutasa. CAT: catalasa. TBARS: sustancias \\
reactivas al ácido tiobarbitúrico
\end{tabular}

La Figura 1, muestra la actividad de las enzimas CAT y SOD y la capacidad antioxidante (medida a través de TBARS) de los sueros de los individuos de estudio, en cada día del microciclo, en esta figura se observan cambios similares en la actividad de las variables entre el GE y GP, excepto que para la actividad CAT no hubo diferencias significativas entre los días de estudio en el GE, y en el GP sí. En la actividad SOD el GE solo presentó un incremento estadísticamente significativo significancia el descenso del día 7 y 14 .

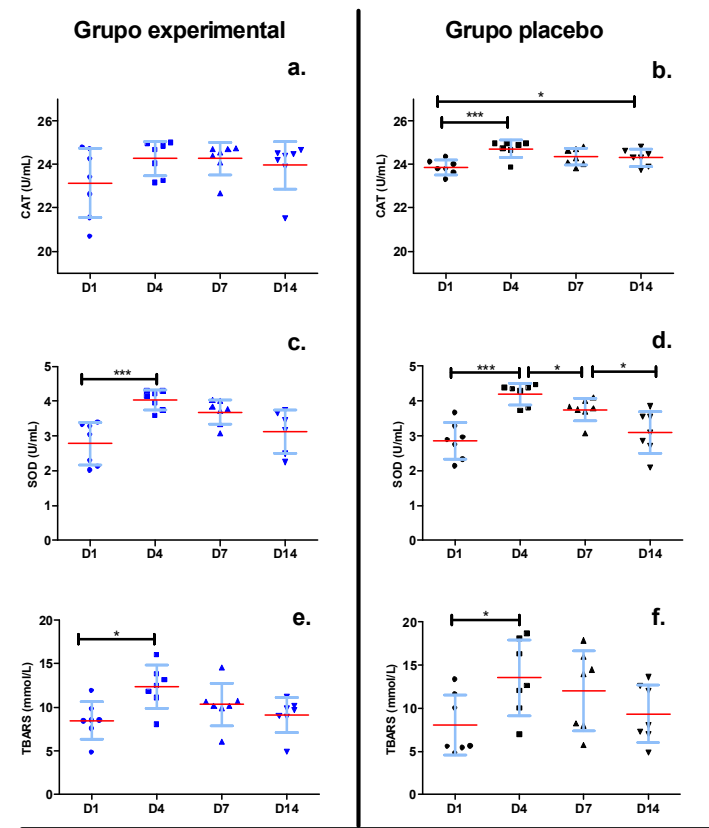

Figura 1. Variables antioxidantes. (a): Catalasa, grupo experimental. (b): Catalasa, grupo placebo. (c): Superóxido dismutasa, grupo experimental. (d): Superóxido dismutasa, grupo placebo. (e): TBARS, grupo experimental. (f): TBARS, grupo placebo. $*: p<.05 . * * *: p<.001$

\section{Discusión}

En este trabajo se estudió el efecto del consumo de Passiflora edulis sobre marcadores bioquímicos (GPT, GOT, CK, Cr, CAT, SOD y TBARS) en mujeres voleibolistas durante un microciclo.

Las estudiantes deportistas, además del estrés que les genera cumplir con los currículos de sus carreras, los cuales incluyen en su mayoría clases magistrales, laboratorios, prácticas, entre otros, también se someten a otros estresores por su entrenamiento físico, técnico y táctico para asumir la competición.

El deporte competitivo, para este caso el voleibol, exige a las atletas someterse a altas cargas de entrena- 
miento y de estrés competitivo, lo que produce cambios tanto en la estructura corporal como en el metabolismo para adaptarse a las exigencias fisiológicas del entrenamiento y la competición (Margaritelis, Paschalis, Theodorou, A., Kyparos \& Nikolaidis, 2020).

Con respecto a las variables antropométricas, la estatura presentó un valor promedio igual al reportado en el año 2017 por García-Cardona et al (García-Cardona, Sánchez-Muñoz, Cabrera-Arismendy \& RestrepoCortés, 2017) en esta misma selección; desde hace varias décadas se ha observado la correlación entre la estatura y el rendimiento deportivo en el voleibol, debido a que la eficiencia en el bloqueo y en las acciones de ataque en este deporte no solo dependen de la capacidad de salto de los deportistas (Fernández, Rubiano \& Hoyos, 2017), sino también de la estatura. Al respecto Fernández, Rubiano \& Hoyos (2017), realizaron una aproximación al perfil morfológico de las voleibolistas de altos logros, identificando como variables determinantes, estatura de $1.82 \mathrm{~m}$, masa de $71.7 \mathrm{~kg}$, porcentaje graso de 17.1\%, envergadura 1.85 m. Así mismo, Malý, Malá, Zahálka, Baláš \& Ėada (2011), mencionan que para la obtención de altos logros el porcentaje adiposo debería estar entre $11,7-27 \%$, y el porcentaje muscular superior al 40\%, lo que muestra que, aunque nuestras voleibolistas se encontraron por debajo de la estatura internacional, se ubican tanto en el porcentaje adiposo como en el muscular en los rangos adecuados específicamente para este deporte.

Este estudio evaluó el efecto del consumo del zumo de $P$. edulis en marcadores bioquímicos de estrés en jugadoras de voleibol. Al respecto la ingesta de nutrientes antioxidantes puede apoyar los sistemas de defensa antioxidantes endógenos de los deportistas, contrarrestando los efectos negativos del daño oxidativo (Buonocore \& Negro, 2015) ocasionado por el entrenamiento; es decir, algunos fitoquímicos presentes en las frutas, juegan un rol importante por su actividad antioxidante, anti-inflamatoria, aumento del potencial inmune, entre otros, específicamente con respecto a la P. edulis, diversos estudios han mostrado que los componentes de diferentes extractos funcionales incluyen fenoles, proteínas, flavonoides, alcaloides, compuestos cianogénicos, glucósidos, vitaminas, minerales y compuestos terpenoides (Gunathilake, Ranaweera \& Rupasinghe, 2018; Rotta, Rodrigues, Cristina, Fontes, Maldaner \& Visentainer, 2019; Septembre-Malaterre, Stanislas, Douraguia \& Gonthier, 2016). La P. edulis es utilizada ampliamente no solo como alimento, sino también en la medicina tradicional, ya que estudios como el de Rojas et al (2009) y Guerrero-Ospina et al (2018) han demostrado como el consumo de jugo de $P$. edulis contribuye en la disminución de la presión arterial en pacientes hipertensos; también estudios in vitro (Aguillón-Osma, Luzardo-Ocampo, Cuellar-Nuñez, Maldonado-Celis, Loango-Chamorro \& Campos-Vega, 2019; Ramirez, Arango, Maldonado, Uribe, Aguillon, Quintero \& Loango, 2019) han demostrado que esta planta es una fuente potencial de compuestos fitoquímicos con propiedades antioxidantes, antiproliferativas, y anticancerígenas para el tratamiento de pacientes con cáncer colorrectal. A pesar de que no se encontró un efecto significativo del consumo de P. edulis, los resultados muestran cambios en el comportamiento de los biomarcadores durante los días evaluados, específicamente las transaminasas (GPT y GOT) que, en el deporte junto con la $\mathrm{CK}$ y la $\mathrm{Cr}$, se utilizan como indicador de daño y destrucción muscular. En nuestro estudio GPT y GOT, se comportaron de manera similar tanto en GE como GP, aumentando significativamente su concentración en D4 con respecto a D1, con posterior descenso en D7 y D14. La concentración de las transaminasas se ve afectada debido a alteraciones de la condición corporal que perturbe el metabolismo de la glucosa, proteínas y lípidos, como lo es el ejercicio físico. Así mismo, el aumento de GPT y GOT se relaciona con el daño a las células del músculo esquelético, y a las del hígado que también sufren una enorme carga de actividad en el ejercicio extenuante ya que es el hígado el que promueve la gluconeogénesis a partir del ácido láctico formado en el músculo (Kobayashi, Suzuki \& Sugai, 2020). Los resultados muestran que en el día 14, las transaminasas no habían regresado a los valores basales del día 1. Al respecto, Pettersson, Hindorf, Persson, Bengtsson, Malmqvist, Werkström \& Ekelund (2007) afirman que el ejercicio muscular intenso puede aumentar la función hepática durante al menos siete días después del ejercicio. En este mismo sentido, Sjogren (2007) indica que las transaminasas pueden permanecer elevadas hasta una semana después de cesar el ejercicio, tal como ocurrió en la presente investigación; más aún Chinedu, Chima, Emmanuel, Nwabunwanne, \& Chinedu (2018) también encontraron una elevación de GPT y otros marcadores de función hepática pero no de GOT en futbolistas sometidos a entrenamiento de $30 \mathrm{minu}-$ tos, tres veces a la semana por tres semanas. El comportamiento de las transaminasas en nuestro estudio es similar a los resultados reportados por (Shin, Park, Ahn, Park \& Kim, 2016) en atletas varones de larga distancia, en donde, GPT mostró un aumento menor en com- 
paración con GOT. Estos hallazgos sugieren que el ejercicio vigoroso como un microciclo de competición afecta los niveles de transaminasas.

Con respecto a la CK sérica se ha estudiado ampliamente y se considera el principal marcador de daño muscular (Santi et al., 2020), además altos niveles de $\mathrm{CK}$ en atletas sanos pueden estar correlacionados con cargas de trabajo de entrenamiento físico (Brancaccio, Maffulli \& Limongelli, 2007). En este sentido, las respuestas de los niveles de CK en los diferentes días evaluados del microciclo en nuestro estudio se ajustaron a lo descrito en la literatura en relación con el aumento de CK y el daño muscular. Comportamiento similares fueron reportados en hombres voleibolistas por Berriel, Rocha, Soares, Schons, Droescher, Peyré-Tartaruga \& Martins (2020), aunque estos autores realizaron un seguimiento por 16 semanas, y en este trabajo solo por 14 días, los resultados muestran en ambos grupos (GE y GP) diferencias significativas en el incremento de $\mathrm{CK}$ en D4 con respecto al D1, y, el no retorno en el D14, a los valores basales del día 1 , esto último posiblemente ocasionado por la fatiga, la cual puede generar un desequilibrio de las bombas $\mathrm{Na}^{+} / \mathrm{K}^{+}$-ATPasa provocando una elevación osmótica, generando daño en las miofribrillas (Allen, 2004). La ingesta del zumo de $P$. edulis no pudo disminuir las concentraciones de $\mathrm{CK}$ en GE comparado con GP, después de los partidos de voleibol, estos resultados también fueron observados en el estudio de Martins et al (2020), en donde evaluaron el efecto del consumo de jugo de uva sobre el estrés oxidativo y la inflamación en jugadores de voleibol masculino; una hipótesis en relación con estos resultados y los nuestros es la posibilidad de una dosis insuficiente.

La concentración de $\mathrm{Cr}$ en este estudio aumentó desde el día 1 al 7 y disminuyó en el día 14, en ambos grupos y sin diferencias significativas al comparar el GE con el GP; durante el microciclo, la Cr aumentó en D4 y D7 comparada con D1 en GE $(29.54 \%$ y $30.68 \%$ respectivamente), al igual que en GP (29.54\% y $39.77 \%$ respectivamente). En ambos grupos la $\mathrm{Cr}$ desciende siete días después del microciclo, sin alcanzar los niveles de inicio, sin embargo, en el GE el descenso fue mayor (27.6\%) comparado con el GP $(25,2 \%)$, estos resultados son concordantes con los reportados por Nowakowska, Kostrzewa-Nowak \& Nowak (2019), en donde muestran variaciones del nivel de $\mathrm{Cr}$ en el tiempo. Así mismo, estudios como el de Jacobs, Sluik, Rokling-Andersen, Anderssen \& Drevon (2009), mencionan que después de un ejercicio de intensidad moderada o alta, hay una disminución en el volumen de orina y una marcada reducción en el flujo de plasma renal y de la tasa de filtración que conducen al aumento de Cr. En este estudio también se observó una disminución con el tiempo de la TFG hasta el día siete, lo que estaría apoyando esta hipótesis.

La TFG basal, para GE y GP, se encontró dentro de los valores de referencia ( 90 a $120 \mathrm{~mL} / \mathrm{min} / 1.73 \mathrm{~m}^{2}$ ) (National Kidney Foundation, 2002), pero la TFG disminuyó desde el día 1 al 7 y aumentó en el día 14, en ambos grupos, sin volver a condiciones basales, y sin diferencias significativas al comparar los grupos, pero al comparar el comportamiento dentro del mismo grupo se encontraron diferencias significativas tanto en GE como en GP entre el día 1 y 4, y el día 7 y 14. La disminución de laTFG, en los días 4 y 7, es dada, por el incremento de la $\mathrm{Cr}$ (Refaie, Moochhala \& Kanagasundaram, 2007).

Como parte de los marcadores bioquímicos de estrés oxidativo en las voleibolistas también se midió SOD, CAT y la capacidad antioxidante medida como TBARS; al respecto, la SOD presentó un mayor incremento en GP $(47.01 \%)$ en D4 con respecto a D1, en comparación con el GE (44.96\%), también, el nivel de actividad de esta enzima disminuyó menos en GE en el D7 $(8,68 \%)$ y D14 (15.21\%) comparado con D4, mientras que en GP, además que la actividad de la enzima subió más en D4, también tuvo una disminución significativa entre los días 7 y 14.

La actividad de la CAT presentó leves incrementos en D4 con respecto al D1 (GE: 4.88\% y GP: 3.61\%), y no desciende significativamente en ambos grupos.

$\mathrm{Al}$ respecto el sistema antioxidante (SOD, CAT y Glutation Peroxidasa) actúa para evitar la acumulación del radical superóxido y el peróxido de hidrógeno (De la Cruz, E., Pino, Moreno, Cañadas \& Ruiz-Risueño, 2008), generados durante el ejercicio. Si bien nuestros resultados muestran cambios en SOD, pero no en CAT, varios estudios indican que los niveles de estas enzimas se expresan de manera diferenciada en diversas células, así, el estudio de Sureda encontró que la actividad de la CAT y la glutatión peroxidasa descienden en neutrófilos, pero permanecen estables en plasma después de un ejercicio extenuante. También nuestros resultados son similares al estudio realizado por Matthaiou et al (2014), en donde evaluaron el efecto antioxidante del consumo de jugo de granada (Punica granatum L.) en humanos, encontrando que a nivel de la CAT no cambió significativamente, aunque a diferencia nuestra, los autores evaluaron la actividad enzimática en eritrocitos.

Con relación a la SOD, aunque estudios como el de 
Ammar et al (2017) con levantadores de pesa élite, muestra que la suplementación con jugo de granada tiene el potencial de atenuar el estrés oxidativo al mejorar las respuestas antioxidantes evaluadas de forma aguda y hasta $48 \mathrm{~h}$ después de una sesión intensiva de entrenamiento de levantamiento de pesas, otros estudios no encontraron cambios en la SOD, tal como lo muestra el trabajo realizado por Sadowska- Krêpa, KBapciñska, Podgórski, Szade, Tyl \& Hadzik (2015), en donde examinó si el consumo regular de una mezcla de jugo a base de baya de açai (Euterpe oleracea Mart.) afectaría el rendimiento del sprint y mejoraría el estado de antioxidantes en sangre y el perfil de lípidos en atletas junior.

Finalmente, en cuanto a los BMEOX, TBARS presentó un menor incremento en GE (45.22\%) con respecto a GP (67.82\%) en D4 al compararlo con D1, presentando también GE (16.38\%) mayor descenso en D7 versus $\mathrm{D} 4$. $\mathrm{Al}$ respecto, resultados similares relacionados a la disminución de TBARS fueron reportados por Sadowska- Krêpa, KBapciñska, Podgórski, Szade, Tyl \& Hadzik (2015) y Martins et al (2020), lo que estaría mostrando una reducción en la peroxidación lipidica.

Los resultados obtenidos en el presente trabajo, indican que si bien el consumo de $2 \mathrm{ml}$ del zumo de $P$. edulis durante 7 días no parece revelar significancias estadísticas en los cambios observados en los biomarcadores evaluados al comparar los dos grupos, si muestran que el consumo de $P$ edulis puede mejorar la capacidad de respuesta al estrés oxidativo de las volibolistas, al disminuir la necesidad de regular al alza las enzimas de defensa antioxidante, como lo ha propuesto Radak, Chung \& Goto (2008); situación similar se presenta con la SOD, en donde en el grupo GE, los cambios no son significativos entre los días del microciclo, mientras en el GP estos cambios entre los dias son significativos. Así, el aumento significativo de CAT y SOD día a día, visto en el GP y no en el GE, podría sustentar esta hipótesis de la regulación a la baja de las enzimas del sistema antioxidante debido al aporte de antioxidantes presentes en el jugo de $P$ edulis, que desestimulan por no ser necesarias posiblemente la expresión o actividad de las enzimas en mención. Sin embargo, esto es expeculativo y se necesita profundizar en el conocimiento y reducir las limitantes del estudio, como son el tamaño de la muestra, el que no se evaluó previamente si la cantidad de jugo era suficiente para mostrar el efecto y control estricto de otros estresores en las deportistas entre otros limitantes.

\section{Conclusiones}

Este estudio muestra que el ejercicio organizado en un microciclo produce cambios día a día en los biomarcadores de estrés oxidativo, aumentando su actividad con el aumento de la intensidad del ejercicio, y estos biomarcadores no retornan a sus niveles basales aun después de siete días de finalizado el microciclo.

El estudio también muestra que el consumo de $P$. edulis no afectó de manera significativa las actividades de SOD y CAT, pero que estas tienen un comportamiento diferente día a día en el grupo que consumió el zumo.

Se recomienda investigar estos biomarcadores en distintos deportes y tipos de mesociclos y evaluar diferentes concentraciones del zumo a nivel basal, previa caracterización bioquímica de sus componentes debido a la variabilidad de su cultivo.

\section{Agradecimientos}

A las deportistas que participaron en el estudio, al entrenador Elber Andrés Acosta, al preparador físico Jhon Iván Robledo, al profesor Oscar Sánchez, y al equipo de trabajo de GIFAS y GECAVYME.

\section{Conflicto de intereses y financiación}

Los autores declaramos que no existen conflicto de interés.

Este proyecto fue financiado por el Departamento Administrativo de Ciencia Tecnología e Innovación COLCIENCIAS, convocatoria 727 de 2015.

\section{Referencias}

Aguillón-Osma, J., Luzardo-Ocampo, I., Cuellar-Nuñez, M. L., Maldonado-Celis, M. E., Loango-Chamorro, N., \& CamposVega, R. (2019). Impact of in vitro gastrointestinal digestion on the bioaccessibility and antioxidant capacity of bioactive compounds from Passion fruit (Passiflora edulis) leaves and juice extracts. Journal of Food Biochemistry, 43(7), 1-11. https:/ /doi.org/10.1111/jfbc. 12879

Allen, D. G. (2004). Skeletal muscle function: Role of ionic changes in fatigue, damage and disease. Clinical and Experimental Pharmacology and Physiology, 31(8), 485-493.https://doi.org/ 10.1111/j.1440-1681.2004.04032.x

Ammar,A.,Turki,M., Hammouda, O., Chtourou, H.,Trabelsi,K., Bouaziz, M.,Abdelkarim, O.,Hoekelmann,A.,Ayadi,F.,Souissi, N., Bailey, S. J.,Driss,T., \&Yaich, S. (2017).Effects of pomegranate juice supplementation on oxidative stress biomarkersfollowing 
weightlifting exercise. Nutrients, 9(8), 1-13.https: / doi.org/ 10.3390/nu9080819

Azizbeigi, K., Stannard, S. R., Atashak, S., \& Mosalman Haghighi, M. (2013).Antioxidant enzymes and oxidative stress adaptation to exercise training: Comparison of endurance, resistance, and concurrent training in untrained males. Journal of Exercise Science and Fitness, 1-6. https://doi.org/10.1016/ j.jesf.2013.12.001

Blázquez, D., \& Hernández, J. (1984). Clasificación o taxonomías deportivas. Inef.

Bouet, M. (1968). Signification du sport. P.U.F.

Brancaccio, P., Maffulli, N., \& Limongelli, F. M. (2007). Creatine kinase monitoring in sport medicine. British Medical Bulletin, 81-82(1), 209-230. https://doi.org/10.1093/bmb/ $\operatorname{ldm} 014$

Buonocore, D. , \& Negro, M. (2015). Anti-inflammatory dietary interventions and supplements to improve Performance during athletic training.J Am Coll Nutr, 34(1), 62-67. https: / / doi.org/10.1080/07315724.2015.1080548

De la Cruz Sánchez, E., Pino Ortega, J., Moreno Conteras, M. I., Cañadas Alonso, M., \& Ruiz-Risueño Abab, J. (2008). Micronutrientes antioxidantes y actividad física: evidencias de las necesidades de ingesta a partir de las nuevas tecnologías de evaluación y estudio del estrés oxidativo en el deporte. Retos, 13, 11-14. https://doi.org/10.47197/retos.v0i13.35021

Dolores, C., Benavides, E., \& Osso, Ó. (2018). Efecto del consumo de una bebida a base de Physalis peruviana, Passiflora edulis, Ananas comosus, Avena sativa, Linum usitatissimum y Stevia rebaudiana, sobre el perfil lipídico y glicemia, de mujeres con sobrepeso y obesidad. Revista de La Sociedad Química Del Perú, 84(1), 107-118. https://doi.org/10.37761/ rsqp.v84i1.172

Estrada-Díaz, S.A., Hernández-Cruz, G., Cruz-Castruita, R. M., Zavala-Flores, L. M., Rodríguez-Bies, E., \& Rangel-Colmenero, B. R. (2017). Estrés oxidativo y capacidad antioxidante en deportistas con dieta rica en antioxidantes con zarzamora (Rubus sp.) Sylvia. Revista de Psicologia Del Deporte, 26(2), 173-179.

Fernández,J. ., Rubiano,P. ., \& Hoyos,L. . (2017).Perfilmorfológico de voleibolistas de altos logros. Revisión. Revista Internacional de Medicina y Ciencias de La Actividad Física y El Deporte, 17(68), 775-794. https://doi.org/10.15366/rimcafd2017.68.012

Fernández, J., Da Silva-Grigoletto, M., \&Túnez-Fiñana, I. (2009). Estrés oxidativo inducido por el ejercicio J.M. RevistaAndaluza de Medicina Del Deporte, 2(1), 19-34.

García-Cardona, D. , Landázuri, P., \& Sánchez-Muñoz, O. (2021). Effect of a shock micro-cycle on biochemical markers in university soccer players. Int. J. Environ. Res. Public Health, 18(3581). https://doi.org/10.3390/ijerph18073581

García-Cardona, D., Sánchez-Muñoz, O., Cabrera-Arismendy, C., \& Restrepo-Cortés, B. (2017). Perfil lipídico, antropométrico y condición física de estudiantes deportistas universitarios. Universidad y Salud, 19(2), 267. https://doi.org/10.22267/ rus. 171902.89

García, M. (2002). Guía Técnica Cultivo de Maracuyá Amarillo.
González, N. F., \& Rivas,A.D. (2018).Actividad física y ejercicio en la mujer. Revista Colombiana de Cardiología, 25(S1), 125-131. https://doi.org/10.1016/j.rccar.2017.12.008

Guerrero-Ospina, J. C., Nieto, O. A., Zarate, P., Loango, N., Restrepo, B., \& Landazuri, P. (2018). Beneficial effects of Passiflora edulis on blood pressure and reduction of oxidative stress. Indian Journal of Science andTechnology, 11(43), 1-8. https:/ /doi.org/10.17485/ijst/2018/v11i43/134064

Gunathilake, K., Ranaweera, K., \& Rupasinghe, H. (2018).Analysis of rutin , b -carotene, and lutein content and evaluation of antioxidant activities of six edible leaves on free radicals and reactive oxygen species. J Food Biochem, 42(e12579), 1-9. https: / / doi.org/10.1111/jfbc. 12579

He, X., Luan, F.,Yang,Y., Wang, Z., Zhao, Z., Fang, J., \&Wang, M. (2020). Passiflora edulis/ : An insight into current researches on phytochemistry and pharmacology. Front Pharmacol, 11(617), 1-16. https://doi.org/10.3389/ fphar.2020.00617

Hu,Y., Jiao, L., Jiang, M.,Yin, S., Dong, P., Zhao, Z., Ho, P., \&Wang, D. (2017). A new C-glycosyl flavone and a new neolignan glycoside from Passiflora edulis Sims peel. Natural Product Research, 6419, 1-7. https://doi.org/10.1080/ 14786419.2017.1410809

Kitada,M., Ogura,Y., Maruki-Uchida,H.,Sai,M., Suzuki,T., Kanasaki, K., Hara,Y., Seto, H., Kuroshima,Y., Monno, I., \& Koya, D. (2017). The effect of piceatannol from passion fruit (Passiflora edulis) seeds on metabolic health in humans. Nutrients, 9(10), 1-17. https://doi.org/10.3390/nu9101142

Kobayashi,A., Suzuki,Y.,\& Sugai,S.(2020). Specificity of transaminase activities in the prediction of drug-induced hepatotoxicity. Journal of Toxicological Sciences, 45(9), 515-537. https://doi.org/ $10.2131 /$ jts. 45.515

Margaritelis, N.V, Paschalis, V., Theodorou, A.A., Kyparos, A., \& Nikolaidis, M. G. (2020). Redox Biology Redox basis of exercise physiology. Redox Biology, 35(101499). https:/ / doi.org/10.1016/j.redox.2020.101499

Martínez-Rodríguez,A., Mira-Alcaraz, J., Cuestas-Calero, B., PérezTurpín, J., \& Alcaraz, P. (2017). La Pliometría en el Voleibol Femenino. Retos, 32, 208-213. https://doi.org/10.47197/ retos.v0i32.56053

Martins, N. C., Dorneles, G. P., Blembeel, A. S., Marinho, J. P., Proença, I. C. T., da Cunha Goulart, M. J.V., Moller, G. B., Marques, E. P., Pochmann, D., Salvador, M., Elsner,V., Peres, A., Dani, C., \& Ribeiro, J. L. (2020). Effects of grape juice consumption on oxidative stress and inflammation in male volleyball players: A randomized, double-blind, placebocontrolled clinical trial. Complementary Therapies in Medicine, 54(102570), 1-9. https://doi.org/10.1016/ j.ctim.2020.102570

Maruki-Uchida,H., Morita, M.,Yonei,Y., \& Sai,M. (2018). Effect of passion fruit seed extract rich in piceatannol on the skin of women:A randomized, placebo-controlled, double-blind trial. Journal of Nutritional Science and Vitaminology, 64(1), 75-80. https: / / doi.org/10.3177/jnsv.64.75

Matveiev, L. (1975). Periodización del entrenamiento deportivo. Inef. 
Molinero, O., Salguero,A., \& Márquez, S. (2012). Estrés-recuperación en deportistas y su relación con los estados de ánimo y las estrategias de afrontamiento. Revista de Psicología Del Deporte, 21(1), 163-170. http://0search.ebscohost.com. library.ucc.ie / login.aspx ?direct $=$ true $\& \mathrm{db}=$ psyh $\& A N=2012-03560$ 020\&site=ehost-live\%5Cnolga.molinero@unileon.es

National Kidney Foundation. (2002). K/DOQI Clinical Practice Guidelines for chronic kidney disease: evaluation, classification, and stratification. Am J Kidney Dis, 39(S1), 266.

Nieman,D. C., Gillitt, N.D., Knab,A.M., Shanely, R.A., Pappan, K. L., Jin, F., \& Lila, M. A. (2013). Influence of a polyphenolenriched protein powder on exercise-induced inflammation and oxidative stress in athletes: A randomized trial using a metabolomics approach. PLoS ONE, 8(8), 1-11. https:// doi.org/10.1371/journal.pone.0072215

Nowakowska, A., Kostrzewa-Nowak, D., \& Nowak, R. (2019). Blood biomarkers of recovery efficiency in soccer players. Int. J.Environ. Res. Public Health, 16(3279), 1-28. https: / / doi.org/ 10.3390/ijerph16183279

Picón-Martínez, M., Chulvi-Medrano, I., Cortell-Tormo, J. M., \& Cardozo, L.A.(2019).La potenciación post-activación en el salto vertical: una revisión (Post-activation potentiation in vertical jump: a review). Retos, 36, 44-51. https://doi.org/ 10.47197/retos.v36i36.66814

Radak,Z., Chung, H.Y., \& Goto, S. (2008). Systemic adaptation to oxidative challenge induced by regular exercise. Free Radical Biology and Medicine, 44(2), 153-159. https://doi.org/ 10.1016/j.freeradbiomed.2007.01.029

Ramirez,V.,Arango, S. S., Maldonado, M. E., Uribe, D., Aguillon, J., Quintero, J. P., \& Loango, N. (2019). Biological activity of Passiflora edulisf. Flavicarpa ethanolic leaves extract on human colonic adenocarcinoma cells. Journal of Applied Pharmaceutical Science, 9(2), 64-71. https://doi.org/10.7324/ JAPS.2019.90209

Refaie, R., \& Moochhala, SH Kanagasundaram, N. (2007). How we estimate GFR-a pitfall of using a serum creatinine-based formula. Clin Nephrol, 68, 235-237.

Reid, M. B. (2008). Free radicals and muscle fatigue/ : Of ROS, canaries, and the IOC. Free Radical Biology \& Medicine, 44, 169-179. j.freeradbiomed.2007.03.002

Reynoso-Sánchez, L.-F., Hernández-Cruz, G., López-Walle, J., Rangel-Colmenero, B., Quezada-Chacón, J.-T., \& Sánchez, J. C. J. (2016). Balance de estrés-recuperación en jugadores universitarios de voleibol durante una temporada. Retos, 30, 193 197.

Rodríguez, M. (2015).Voleibol: análisis de su estructura y características para entender el juego. Efdeportes, Revista Digital, 20(210), 1-6. https://doi.org/10.1016/B978-0-32360984-5.00062-7

Rodriguez, Q. (2015).Voleibol: análisis de su estructura y características para entender el juego. EFDeportes.Com, Revista Digital, 20(210), 1-6.

Rotta, E., Rodrigues, C., Cristina, I., Fontes, S., Maldaner, L., \&
Visentainer,J. (2019). Determination of phenolic compounds and antioxidant activity in passion fruit pulp (Passiflora spp.) using a modified QuEChERS method and UHPLC-MS/ MS. LWT - Food Science and Technology, 100, 397-403. https: / / doi.org/10.1016/j.lwt.2018.10.052

Sánchez-Moreno, M., García-Asencio, C., González-Badillo, J. J., \& Díaz-Cueli, D. (2018). Strength and vertical jump performance changes in elite male volleyball players during the season. Retos, 34, 291-294. https://doi.org/10.47197/ retos.v0i34.65898

Santi, M. C., Galán, B. S. M., Terrazas, S. I. M., De Carvalho, F. G., Vieira, T. S., Silveira, G. C., Deminice, R., \& de Freitas, E. C. (2020). Effect of creatine supplementation on muscle damage markers and physical performance in volleyball athletes. Cultura, Ciencia y Deporte, 15(45), 377-385. https://doi.org/ 10.12800/ccd.v15i45.1515

Septembre-Malaterre,A., Stanislas, G., Douraguia, E., \& Gonthier, M. (2016). Evaluation of nutritional and antioxidant properties of the tropical fruits banana, litchi , mango , papaya , passion fruit and pineapple cultivated in Réunion French Island. Food Chemistry, 212, 225-233. https://doi.org/10.1016/ j.foodchem.2016.05.147

Shin, K. A., Park, K. D., Ahn, J., Park, Y., \& Kim, Y. J. (2016). Comparison of changes in biochemical markers for skeletal muscles, hepatic metabolism, and renal function after three types of long-distance running. Medicine (United States), 95(20), 1-6. https: / / doi.org/10.1097/MD.0000000000003657 Silva, R. O., Damasceno, S. R. B., Brito,T.V., Dias, J. M., Fontenele, A. M., Braúna, I.S., Júnior, J. S. C., Maciel, J. S., De Paula, R. C. M., Ribeiro, R.A., Souza, M.H.L.P., Freitas,A.L.P., Medeiros, J.V.R., Silva,D. C., \& Barbosa,A.L. R. (2015). Polysaccharide fraction isolated from Passiflora edulis inhibits the inflammatory response and the oxidative stress in mice. Journal of Pharmacy and Pharmacology, 67(7), 1017-1027. https://doi.org/ 10.1111/jphp.12399

Vilela, G., Caniuqueo-Vargas, A., Ramirez-Campillo, R., Hernández-Mosqueira, C., \& Da Silva, S. F. (2021). Efecto del entrenamiento pliométrico en la fuerza explosiva de niñas puberes practicantes de voleibol. Retos, 40, 41-46. https: / / doi.org/10.47197/retos.v1i40.77666

Vitón, A. (2012). La caracterización del juego de voleibol de sala contemporáneo. Efdeportes, Revista Digital, 17(168), 1-4.

Zeraik, M., Serteyn, D., Deby-Dupont, G., Wauters, J.-N.,Tits, M., Yariwake, J., Angenot, L., \& Franck, T. (2011). Evaluation of the antioxidant activity of passion fruit (Passiflora edulis and Passiflora alata ) extracts on stimulated neutrophils and myeloperoxidase activity assays. Food Chemistry, 128(2), 259 265. https://doi.org/10.1016/j.foodchem.2011.03.001 Zhang,Y.J., Zhou,T.,Wang, F., Zhou,Y., Li,Y., Zhang, J.J., Zheng, J., Xu, D. P., \& Li, H. Bin. (2016). The effects of syzygium samarangense, passiflora edulis and solanum muricatum on alcohol-induced liver injury. International journal of Molecular Sciences, 17(10), 1-11. https://doi.org/10.3390/ ijms17101616 